\title{
DOENÇA RENAL CRÔNICA: EXPLORANDO NOVAS ESTRATÉGIAS DE COMUNICAÇÃO PARA PROMOÇÃO DA SAÚDE
}

\section{Chronic Kidney Disease: exploring new strategies of communication for health promotion}

\section{Enfermedad renal crónica: explorando nuevas estrategias de comunicación para la promoción de la salud}

\author{
Juliana Gomes Ramalho de Oliveira \\ Universidade de Fortaleza - UNIFOR - Fortaleza (CE) - Brasil \\ Geraldo Bezerra da Silva Júnior \\ Universidade de Fortaleza - UNIFOR - Fortaleza (CE) - Brasil \\ José Eurico Vasconcelos Filho \\ Universidade de Fortaleza - UNIFOR - Fortaleza (CE) - Brasil
}

\section{RESUMO}

Objetivo: Descrever o desenvolvimento de novas estratégias de comunicação para promoção da saúde no âmbito da doença renal crônica (DRC). Métodos: Pesquisa de natureza aplicada, pois objetiva a produção de conhecimentos direcionados à solução de problemas específicos por meio de ferramentas práticas e da análise das hipóteses levantadas. As ferramentas foram desenvolvidas pela parceria entre o Programa de Pós-Graduação em Saúde Coletiva e o Núcleo de Aplicação em Tecnologia da Informação da Universidade de Fortaleza. O estudo intitulado "Renal Health" foi dividido em três fases consecutivas: Fase 1 - Desenvolvimento do aplicativo Renal Health; Fase 2 - Construção de conteúdos e plataformas digitais vinculadas ao aplicativo; e Fase 3 - Teste de impacto clínico e adesão ao aplicativo. No presente momento, o estudo encontra-se com a fase 1 concluída e com a fase 2 em andamento. Resultados: Foi desenvolvido o aplicativo Renal Health para uso em dispositivos móveis, em plataforma Android, com interfaces para a população geral, ou seja, pessoas que não fazem tratamento para DRC, pacientes renais crônicos em hemodiálise e transplantados renais. A fase 2 do estudo encontra-se em desenvolvimento, ocorrendo por meio de reuniões entre as equipes de pesquisadores e dos setores saúde e tecnologia para a compilação dos conteúdos e delineamento do projeto gráfico das plataformas digitais. Conclusão: A comunicação entre os profissionais de saúde e os portadores de DRC é passível de contínuo aperfeiçoamento, dada a variedade das modalidades de tratamento e suas peculiaridades. $O$ aplicativo Renal Health e as plataformas digitais se propõem a apoiar ambos nesse processo.

Descritores: Insuficiência Renal Crônica; Diálise Renal; Transplante de Rim; Meios de Comunicação.

\section{ABSTRACT}

Objective: To describe the development of new communication strategies for health promotion in the context of chronic kidney disease (CKD). Methods: This is an applied research aimed at producing knowledge to solve specific problems through practical tools and analysis of hypotheses. The tools were developed through a partnership between the Public Health Graduate Program and the Center of Applied Information Technology of the University of Fortaleza. The study titled "Renal Health" was divided into three consecutive phases: Phase 1 - Development of the Renal Health application; Phase 2 - Elaboration of digital content and platforms linked to the application; and Phase 3 - Tests of clinical impact and adherence to the application. Phase 1 was finished and phase 2 is currently ongoing. Results: The Renal Health application was developed to be used in Android mobile phones and features interfaces targeted at the general population, i.e., people who do not receive CKD treatment, CKD patients undergoing hemodialysis and kidney transplant patients. Phase 2 of the study is being carried out through meetings between researchers and health and technology teams to develop the content and design the graphical project of the digital platforms. Conclusion: Communication between health professionals and CKD patients is subjected to continuous improvement given the 
variability of treatment modalities and its peculiarities. The Renal Health application and digital platforms intend to support both professionals and patients in this process.

Descriptors: Renal Insufficiency, Chronic; Renal Dialysis; Kidney Transplantation; Communications Media.

\section{RESUMEN}

Objetivo: Describir el desarrollo de nuevas estrategias de comunicación para la promoción de la salud en el ámbito de la Enfermedad Renal Crónica (ERC). Métodos: Investigación de naturaleza aplicada pues tiene el objetivo de producir conocimientos dirigidos para la solución de problemas específicos a través de herramientas prácticas y del análisis de las hipótesis presentadas. Las herramientas han sido desarrolladas con la colaboración entre el Programa de Pos-Grado de Salud Colectiva y el Núcleo de Aplicación en Tecnología de la Información de la Universidad de Fortaleza. El estudio intitulado "Renal Health" ha sido dividido en tres fases consecutivas: Fase 1 - Desarrollo del aplicativo Renal Health; Fase 2 - Construcción de contenidos y plataformas digitales vinculadas al aplicativo; y Fase 3 - Prueba de impacto clínico y adhesión al uso del aplicativo. En el momento el estudio está con la fase 1 concluida y la fase 2 en seguimiento. Resultados: Ha sido desarrollado el aplicativo Renal Health para dispositivos móviles, en la plataforma Android, con interfaces para la población general, o sea, personas que no tiene el tratamiento para la ERC, pacientes renales crónicos en hemodiálisis y trasplantados renales. Se está desarrollando la fase 2 del estudio con reuniones entre los equipos de investigadores y los sectores de salud y tecnología para la compilación de los contenidos y delineamiento del proyecto gráfico de las plataformas digitales. Conclusión: La comunicación entre los profesionales sanitarios y los portadores de ERC es pasible de perfeccionamiento continuo por la variedad de modalidades de tratamiento $y$ sus peculiaridades. El aplicativo Renal Health y las plataformas digitales se proponen a apoyarse en ese proceso.

Descriptores: Insuficiencia Renal Crónica; Diálisis Renal; Trasplante de Riñón; Medios de Comunicación.

\section{INTRODUÇÃO}

A doença renal crônica (DRC) vem ganhando destaque no mundo todo devido ao crescimento exponencial observado nas últimas décadas e ao elevado custo do seu tratamento. Definida como a presença de danos nos rins ou a diminuição da função renal, durante três meses ou mais, com repercussões no estado geral do paciente ${ }^{(1)}$, a DRC tem como principais causas a hipertensão arterial sistêmica (HAS) (35\%) e a diabetes mellitus (DM) $(29 \%)^{(2)}$, ambos importantes problemas de saúde pública, com grande impacto no perfil de morbimortalidade dos portadores. Como as chances de desenvolver DRC aumentam com a idade, o envelhecimento populacional representa um risco global.

No Brasil, os números alarmantes da DRC podem ser confirmados por meio dos levantamentos anuais realizados pela Sociedade Brasileira de Nefrologia (SBN). Os resultados mostram que, enquanto nos anos 2000 havia aproximadamente 42.695 pacientes em diálise no país, em 2017 já eram 126.583, expondo o comportamento progressivo da doença ao longo dos últimos anos. Observa-se também que, embora a DRC tenha uma estreita relação com o envelhecimento, o número de pessoas acometidas a partir da faixa etária de 20 a 44 anos, ou seja, no auge da idade economicamente ativa, é preocupante ${ }^{(3)}$. Estudos comprovam que o diagnóstico precoce e a preservação da função renal são as estratégias mais eficazes para a prevenção da $D R C^{(4)}$.

Diante desse contexto, percebe-se um aumento nas iniciativas mundiais para o monitoramento da doença apoiadas em três principais estratégias: a sensibilização, a conscientização e a disseminação do conhecimento sobre a doença, seus fatores de risco e suas complicações ${ }^{(5)}$. Pesquisadores apontam que indivíduos acometidos, ou com histórico familiar de DRC, precisam ser alertados sobre os riscos para sua saúde renal e que o encaminhamento tardio ao nefrologista, ou seja, quando a doença já se encontra em estágio avançado, relaciona-se a maior mortalidade dos pacientes em diálise ${ }^{(6)}$.

Visando a redução da incidência e das complicações associadas à DRC, uma estratégia ainda pouco explorada é o alinhamento das ações de educação e promoção da saúde ao efervescente crescimento tecnológico. Tendo em vista o acesso irrestrito da população aos smartphones e seu uso predominante para o acesso à internet, criam-se canais de comunicação, entre prestadores de assistência à saúde e os usuários da saúde, que podem ser utilizados para o fornecimento de informações e cuidados mais direcionados às necessidades específicas. De acordo com a Pesquisa Nacional por Amostra de Domicílios (PNAD), no ano de 2014, pela primeira vez, o uso de telefone celular para acessar a internet ultrapassou o uso de microcomputadores nos domicílios brasileiros. Em 2015, esse cenário se repetiu e alcançou todas as regiões do país ${ }^{(7)}$. Em 2010, pesquisadores americanos já sinalizavam para as mudanças que o uso dos smartphones estavam causando nos cuidados em saúde, na perspectiva de pacientes e profissionais ${ }^{(8)}$. 
Diante da alta capilaridade tecnológica, surgiu a mHealth, definida pela Organização Mundial da Saúde (OMS) como o uso de tecnologias móveis e sem fio para apoiar a consecução dos objetivos de saúde ${ }^{(9)}$. De acordo com Levine et al (2015), "um dos aspectos mais promissores da mHealth é o seu potencial para melhorar a integração inteligente entre os serviços de saúde e a continuidade dos cuidados, disponibilizando informações no lugar certo e no momento certo"(10). Trata-se de um vasto campo de possibilidades de ações de comunicação para promoção da saúde, em que o usuário é capacitado a fazer escolhas mais saudáveis, por meio do livre acesso às informações de saúde, e pode ser habilitado a realizar o acompanhamento, em tempo real, do seu estado de saúde, utilizando ferramentas tecnológicas de automonitoramento.

Na perspectiva epidemiológica atual de crescimento das doenças crônicas, como a DRC, investir em prevenção e controle por meio de ferramentas que consigam superar as dificuldades de acesso da população aos serviços de saúde e ao mesmo tempo reduzir a demanda por cuidados, pode representar um novo conceito de assistência à saúde no país. Portanto, o objetivo do estudo foi descrever o desenvolvimento de novas estratégias de comunicação para promoção da saúde no âmbito da DRC.

\section{MÉTODOS}

Pesquisa de natureza aplicada, pois objetiva a produção de conhecimentos direcionados à solução de problemas específicos por meio de ferramentas práticas e a análise das hipóteses levantadas. O estudo intitulado "Projeto Renal Health" teve início em maio de 2015 e foi dividido em três fases consecutivas: Fase 1 - Desenvolvimento do aplicativo Renal Health; Fase 2 - Ensino a distância (EAD) com a construção de conteúdos e plataformas digitais vinculadas ao aplicativo; e Fase 3 - Teste de impacto clínico e adesão ao aplicativo. O estudo é desenvolvido em parceira com o Núcleo de Aplicação em Tecnologia da Informação (NATI) da Universidade de Fortaleza; e conta com financiamento da International Society of Nephrology (ISN), por meio do Clinical Research Program, e com o apoio da Sociedade Brasileira de Nefrologia (SBN). Atualmente, encontra-se com a fase 1 concluída e a fase $2 \mathrm{em}$ andamento.

A população do estudo são todas as pessoas, portadoras ou não de DRC, e os profissionais de saúde.

$\mathrm{Na}$ fase 1, utilizou-se como metodologia de desenvolvimento o processo de design centrado no usuário cíclico preconizado e adaptado de Preece et al. (2013), que engloba as atividades primordiais do processo de software: levantamento dos requisitos, especificação/design, construção e avaliações contínuas com a participação dos usuários do software. As etapas da pesquisa foram: levantamento de dados, concepção e desenvolvimento do artefato tecnológico (aplicativo) e avaliação ${ }^{(11)}$.

A fase 2 do estudo, o EAD, conta com o desenvolvimento de uma abordagem em saúde baseada na criação de conteúdos digitais interativos e lúdicos aplicados à internet, às redes sociais, e tecnologias móveis para ampliar o conhecimento da população acerca da DRC, auxiliar os pacientes renais crônicos no tratamento e contribuir com os profissionais de saúde no processo contínuo de educação em saúde. O método de construção dos conteúdos é pautado na literatura e em guidelines mais atualizados dentro da nefrologia. As etapas são: planejamento, implementação e avaliação dos resultados.

A fase 3 está em estágio de delineamento metodológico e envolve estudos longitudinais para avaliação do impacto do uso do aplicativo Renal Health nos resultados em saúde dos usuários, bem como a adesão dos pacientes renais crônicos a esse tipo de estratégia.

O estudo tem como base os princípios legais e éticos que envolvem a pesquisa com seres humanos estabelecidos na Resolução 466/2012, do Conselho Nacional de Saúde ${ }^{(12)}$, sendo submetido e aprovado pelo Comitê de Ética em Pesquisa com Seres Humanos da Universidade de Fortaleza (Parecer $\left.n^{\circ} 1.482 .270 / 2016\right)$.

\section{RESULTADOS}

Na fase 1 da pesquisa, foi desenvolvido o aplicativo Renal Health para uso em dispositivos móveis, em plataforma Android, e uma ferramenta administrativa na web em plataforma JAVA, com interfaces para a população geral, ou seja, pessoas que não fazem tratamento para DRC, pacientes renais crônicos em hemodiálise e transplantados renais (Tabela I). Para embasar a concepção, foram realizadas entrevistas com esses grupos sobre o conhecimento acerca da DRC, dificuldades no tratamento e dúvidas relacionadas à doença.

Originou-se, então, a versão interativa do aplicativo Renal Health, com telas que atendessem às demandas de informação dos usuários e ao layout, ou seja, que proporcionasse melhor compreensão e facilidade de manuseio da ferramenta. A população em geral tem à disposição no aplicativo conteúdo informativo sobre a DRC e a opção da realização de testes, com ou sem resultados de exames, para determinação do risco para a DRC. 
Na sessão para pacientes em HD e transplantados renais, foram desenvolvidas funcionalidades direcionadas ao contexto específico de cada modalidade de tratamento. $O$ aplicativo oferece, ainda, a opção de sincronização via Bluetooth, com sensores visando automatizar a coleta de algumas informações. Foi realizada a integração do aplicativo com uma smartband (pulseira inteligente) que permite a coleta da frequência cardíaca e qualidade do sono. Outros sensores estão sendo testados para integrarem as futuras versões do aplicativo.

Tabela I - Sessões do aplicativo Renal Health de acordo com o público alvo. Fortaleza, Ceará, 2017.

\begin{tabular}{|c|c|c|}
\hline Público alvo & Sessões & Especificações \\
\hline \multirow[t]{2}{*}{ População geral } & Informações & $\begin{array}{l}\text { Funcionamento dos rins: } \\
\text { O que são os rins } \\
\text { Quais as principais funções dos rins } \\
\text { O que é a DRC } \\
\text { Principais causas } \\
\text { Principais sintomas } \\
\text { Tratamento } \\
\text { Como prevenir a DRC } \\
\text { Dúvidas frequentes }\end{array}$ \\
\hline & Testes & $\begin{array}{l}\text { Sem exames: } \\
\text { Teste de hidratação } \\
\text { Questionário de risco para a DRC } \\
\text { Com exames: } \\
\text { Teste da taxa de filtração glomerular }\end{array}$ \\
\hline \multirow[t]{4}{*}{ Pacientes em hemodiálise } & Seu tratamento & $\begin{array}{l}\text { Controle de líquidos } \\
\text { Controle de peso } \\
\text { Exames } \\
\text { Histórico }\end{array}$ \\
\hline & Agenda & $\begin{array}{l}\text { Medicações } \\
\text { Exames } \\
\text { Consultas }\end{array}$ \\
\hline & Informações & $\begin{array}{l}\text { Gerais } \\
\text { Sobre seu tratamento } \\
\text { Fique atento }\end{array}$ \\
\hline & Tabela Nutricional & $\begin{array}{l}\text { Quantidade de fósforo nos alimentos } \\
\text { Quantidade de potássio nos alimentos } \\
\text { Quantidade de sódio nos alimentos }\end{array}$ \\
\hline \multirow[t]{4}{*}{ Pacientes transplantados renais } & Histórico & $\begin{array}{l}\text { Creatinina } \\
\text { Pressão arterial } \\
\text { Glicemia } \\
\text { Peso }\end{array}$ \\
\hline & Agenda & $\begin{array}{l}\text { Medicações } \\
\text { Exames } \\
\text { Consultas }\end{array}$ \\
\hline & Informações & $\begin{array}{l}\text { Fique Atento: } \\
\text { Sinais e sintomas } \\
\text { Recomendações: } \\
\text { Sobre os medicamentos } \\
\text { Gerais } \\
\text { Nutricionais }\end{array}$ \\
\hline & & Dúvidas frequentes \\
\hline
\end{tabular}


Após a finalização da versão piloto do aplicativo, realizou-se um teste de usabilidade com 10 pacientes em hemodiálise. O teste consistia em realizar sete tarefas de maior relevância no aplicativo e preencher um formulário com as impressões sobre o software e questões sobre o grau de dificuldade das tarefas realizadas. A maioria dos pacientes atribuiu valor de "normal", "fácil" e "muito fácil" às questões. A seguir, implementou-se um teste com especialistas em Nefrologia, todos em atuação na área há mais de 5 anos. Os convidados foram orientados a realizar o login no aplicativo para a avaliação e, em seguida, responderem a um formulário sobre os objetivos, estrutura, funcionalidade e relevância do aplicativo. O percentual de aprovação das sentenças foi de $89,6 \%$, e as principais sugestões para ajustes foram: a possibilidade de ampliar a letra ou o tamanho da tela, devido ao comprometimento visual apresentado por muitos pacientes; a redução dos textos e adequações de conteúdo para melhorar a compreensão dos usuários.

A fase 2 do estudo encontra-se na etapa de implementação, que ocorre por meio de reuniões periódicas entre as equipes de pesquisadores para a compilação dos conteúdos relacionados à $\mathrm{DRC}$ e ao delineamento do projeto gráfico das plataformas digitais. A equipe multidisciplinar responsável por essa fase é formada por professores e alunos, de graduação e pós-graduação, das áreas da saúde, enfermagem e medicina, comunicação social, audiovisual e engenharia da computação.

Durante o planejamento, estabeleceu-se a ordem cronológica das plataformas digitais que seriam exploradas. Inicialmente, optou-se pelo desenvolvimento de vídeos informativos sobre a DRC em um canal no YouTube, site americano de compartilhamento de vídeos, o qual tem no Brasil seu segundo maior mercado mundial em horas assistidas, além de possuir cerca de 98 milhões de cidadãos conectados, de acordo com estimativas. Posteriormente, foram criados perfis nas redes sociais Instagram e Facebook para atingir um número ainda maior de pessoas.

A equipe traçou as metas dessa etapa a partir da identificação das seguintes necessidades:

- Construção de conteúdos básicos e compactos sobre a DRC, com linguagem acessível a pessoas dos mais diversos níveis educacionais;

- Interface com o aplicativo Renal Health;

- Divulgação do aplicativo;

- Desenvolvimento de material direcionado aos profissionais de saúde sobre a DRC e o uso do aplicativo da prática assistencial.

Em seguida, foram estabelecidos os requisitos tecnológicos necessários para atender às necessidades, tais como: escolha do modelo audiovisual e da diagramação, que deveria ser, ao mesmo tempo, explicativa e atraente; padronização do design a ser adotado na série de vídeos e o delineamento do projeto gráfico das plataformas digitais.

A construção dos vídeos, quanto aos elementos gráficos que integrariam os cenários, a escolha da paleta de cores e a caracterização da personagem principal foi conduzida pelo grupo de tecnologia da informação e comunicação social. Ao grupo da saúde coube a seleção dos temas de cada vídeo e a síntese lógica do conteúdo com adequação à duração de cada vídeo.

Em consecutivas reuniões, foram apresentadas propostas de layout, às quais foram incorporadas sugestões de remodelagem até a aprovação final por toda a equipe. A identidade visual foi finalizada com a eleição da personagem Kidney (que significa rim em português), um nefrologista que responde às perguntas realizadas por uma voz feminina.

Definidos o conteúdo e o design gráfico, iniciou-se, efetivamente, a produção dos vídeos, com a sincronização e articulação fonética das personagens e a inserção de elementos iconográficos para ajudar a manter a atenção dos espectadores e facilitar a compreensão dos temas. Posteriormente, foi incorporada à equipe um grupo de locutores, conferindo identidade sonora às personagens, e um intérprete de Libras (Língua Brasileira de Sinais) para atender também à demanda de deficientes auditivos e ampliar o alcance das informações.

Para abrigar os vídeos elaborados, foi criado o canal no YouTube chamado Renal Health (https://www.youtube. com/channel/UC3-GHeHAndcrRmbE4I_qE_w) e um perfil na rede social Instagram (https://www.instagram.com/ renal_health/) para a divulgação dos conteúdos compilados. Além disso, em breve será construída uma página no Facebook.

A fase 3 , o estudo longitudinal, com início em setembro de 2018, terá previsão de acompanhamento dos pacientes em hemodiálise e transplantados renais que fazem uso do aplicativo por 2 anos.

\section{DISCUSSÃO}

Pautado em seus objetivos principais, de contribuir na prevenção e adesão ao tratamento da DRC, o Projeto Renal Health tem desenvolvido estratégias inovadoras de comunicação e promoção da saúde, acompanhando a tendência de crescente perfusão da tecnologia da informação na atual configuração social. 
Como estratégia de contenção do avanço da DRC no país, evidenciado pelo aumento do número de pacientes em tratamento dialítico de aproximadamente $300 \%$ em 17 anos, o desenvolvimento de um aplicativo e a elaboração de material educacional digital, voltados ao esclarecimento sobre as doenças renais e disponibilizados gratuitamente na internet, em diferentes plataformas, podem ampliar o conhecimento da população geral e aumentar as chances da incorporação de estilos de vida mais saudáveis como forma de prevenção. Pesquisadores alertam que, para evitar a instalação da doença, o diagnóstico precoce dos grupos de risco e a preservação da função renal são as estratégias mais eficazes ${ }^{(4)}$. Além disso, a ampliação do acesso ao tratamento adequado pode alterar a história natural da doença e reduzir significativamente os custos envolvidos ${ }^{(13)}$.

Tendo em vista que um dos maiores desafios para as equipes de saúde no cuidado ao paciente renal crônico é a adesão ao tratamento ${ }^{(14-17)}$, e ainda que a complexidade da DRC e do tratamento exigem alto nível de envolvimento do paciente e habilidades de autocuidado ${ }^{(18)}$, ferramentas que o ajudem a compreender melhor a doença, conhecer as repercussões dos seus hábitos no estado de saúde e embasem a tomada consciente de decisão podem contribuir em maior adesão.

No cenário brasileiro, a incorporação do método de disseminação de conteúdos a distância tem, ainda, objetivos mais amplos, como contornar o analfabetismo literal e o funcional, dada a transmissão por meio de áudios. Em 2016, a taxa de analfabetismo das pessoas com idade igual ou superior a 15 anos no país foi estimada em $7,2 \%$ $(11,8 \text { milhões de analfabetos })^{(19)}$. Portanto, a adoção de estratégias inovadoras de comunicação em saúde não pode desconsiderar esse grupo.

Vários estudos vêm sendo desenvolvidos ao redor do mundo sobre a usabilidade, implementação e repercussões das abordagens mHealth nos desfechos de diversas condições de saúde, inclusive da DRC ${ }^{(20-22)}$. Revisões sistemáticas destacam o potencial de benefícios clínicos dessas intervenções, mas, ao mesmo tempo, alertam sobre a necessidade de estudos comprobatórios ${ }^{(23,24)}$.

Portanto, acredita-se que, além de desenvolver esses modelos de comunicação e promoção da saúde, faz-se mandatória a mensuração da adesão, alcance e resultados em saúde do uso dessas ferramentas. Como se trata de um campo novo de atuação, no que tange ao emprego da tecnologia da informação e comunicação, principalmente junto aos portadores de DRC, essa análise é fundamental para embasar novos estudos e nortear ações futuras de promoção da saúde.

Por essas razões, a fase 3 deste estudo visa o acompanhamento dos pacientes renais crônicos que fazem uso do aplicativo Renal Health, realizando o registro periódico dos resultados dos exames e intercorrências, bem como entrevistando-os para conhecer as suas percepções sobre a utilização e as contribuições da ferramenta no contexto da doença.

Nesse novo cenário, delineiam-se os estudos denominados híbridos, pois se propõem a avaliar a eficácia e a implementação de uma estratégia terapêutica. Esses estudos adotam uma das seguintes abordagens: teste dos efeitos de uma intervenção clínica nos resultados, com observação e coleta de dados sobre a implementação; e teste simultâneo da intervenção e da implementação, ou teste de uma estratégia de implementação, com observação e coleta de dados sobre o impacto da intervenção nos resultados ${ }^{(25)}$.

Apesar do seu caráter inovador, este experimento tem como limitações a exclusão de uma parcela da população que ainda não tem acesso à internet. Entretanto, é possível estimar que, havendo a manutenção da tendência crescente observada nos últimos anos, esse grupo será cada vez mais reduzido.

Nessas circunstâncias, é importante mencionar que a era tecnológica é uma realidade. Não há como retroceder ou ignorá-la. O grande desafio para a atual geração de profissionais de saúde é agregar a diversidade presente no setor da tecnologia da informação e comunicação às ações de promoção da saúde, aumentando o espectro de possibilidades de atuação e alcançando, simultaneamente, um número incontável de pessoas. Observa-se, nos dias atuais, um período de mudança nos rumos das práticas de assistência à saúde.

\section{CONCLUSÃO}

A comunicação entre os profissionais de saúde e os portadores de DRC é passível de contínuo aperfeiçoamento, dada a variedade das modalidades de tratamento e suas peculiaridades. As novas estratégias, como o aplicativo Renal Health e os espaços criados em plataformas digitais, propõem-se a apoiar ambos nesse processo.

Baseadas em uma abordagem inovadora e diversificada, tendo como público-alvo todas as pessoas, diagnosticadas ou não, essas ações contribuem na difusão do conhecimento sobre a DRC, constituindo-se em estratégia de redução do seu avanço e até mesmo das taxas de mortalidade atribuídas à doença. Para os profissionais, essas 
ferramentas surgem como auxiliares na educação permanente e, para os pacientes, surgem como facilitadores do aprendizado e automonitoramento.

\section{AGRADECIMENTOS}

À International Society of Nephrology (ISN), à Sociedade Brasileira de Nefrologia (SBN), ao Conselho Nacional de Desenvolvimento Científico e Tecnológico (CNPQ), à Fundação Cearense de Apoio ao Desenvolvimento Científico e Tecnológico (Funcap) e à Fundação Edson Queiroz/Universidade de Fortaleza - UNIFOR pelo apoio e parcerias na construção deste estudo.

\section{CONFLITOS DE INTERESSE}

Os autores declaram que não há conflitos de interesse.

\section{REFERÊNCIAS}

1. Kidney disease. Improving global outcomes. KDIGO 2012 clinical practice guideline for the evaluation and management of chronic kidney disease. Kidney Int Suppl. 2013;3(1):1-163.

2. Sesso RC, Lopes AA, Thomé FS, Lugon JR, Martins CT. Inquérito brasileiro de diálise crônica 2014. J Bras Nefrol. 2016;38(1):54-61.

3. Sociedade Brasileira de Nefrologia. Censo 2017. [Internet]. [acesso em 2018 Jun 26]. Disponível em: http:// www.censo-sbn.org.br/censosAnteriores

4. Kirsztajn GM, Bastos MG. Um chamado à prevenção. J Bras Nefrol. 2015;37(3):285-286.

5. Moura L, Andrade SSCA, Malta DC, Pereira CA, Passos JEF. Prevalência de autorrelato de diagnóstico médico de doença renal crônica no Brasil: Pesquisa Nacional de Saúde, 2013. Rev Bras Epidemiol. 2015;18(Supl 2):181-91.

6. Diegoli H, Silva MCG, Machado DSB, Cruz CERN. Encaminhamento tardio ao nefrologista e a associação com mortalidade em pacientes em hemodiálise. J Bras Nefrol. 2015;37(1):32-7.

7. Ministério do Planejamento, Desenvolvimento e Gestão (BR), Instituto Brasileiro de Geografia e Estatística. Pesquisa nacional por amostra de domicílios: acesso à internet e à televisão e posse de telefone móvel celular para uso pessoal 2015. Rio de Janeiro: IBGE, 2016.

8. California Healthcare Foundation. How smartphones are changing health care for consumers and providers. 2010 [Internet]. [acesso em 2017 Out 15]. Disponível em: https://www.chcf.org/publication/how-smartphonesare-changing-health-care-for-consumers-and-providers/

9. World Health Organization. Global observatory for ehealth series. MHealth: new horizons for health through mobile technologies: second global survey on eHealth [Internet]. [acesso em 2015 Dez 15]. Disponível em: http://www.who.int/goe/publications/goe_mhealth_web.pdf

10. United States Agency for International Development. MHealth Compendium: volume 5. Arlington (VA): African Strategies for Health, Management Sciences for Health; 2015.

11. Preece J, Rogers $\mathrm{Y}$, Sharp H. Design de Interação: além da interação homem-computador. $3^{\mathrm{a}}$ ed. Porto Alegre: Bookman; 2013.

12. Conselho Nacional de Saúde (BR). Resolução 510, de 07 de Abril de 2016 [Internet]. [acesso em: 2017 Set 30]. Disponível em: http://conselho.saude.gov.br/resolucoes/2016/Reso510.pdf

13. Wouters OJ, O'Donoghue DJ, Ritchie J, Kanavos PG, Narva AS. Early chronic kidney disease: diagnosis, management and models of care. Nat Rev Nephrol. 2015;11(8):491-502.

14. Costa-Requena G, Cantarell MC, Moreso F, Parramon G, Seron D. Adherencia al tratamiento tras trasplante renal como indicador de calidad de la información recibida: estudio longitudinal con un seguimiento de 2 años. Rev Calid Asist. 2017;32(1):33-9. 
15. Ibrahim S, Hossam M, Belal D. Study of non-compliance among chronic hemodialysis patients and its impact on patients' outcomes. Saudi J Kidney Dis Transpl. 2015;26(2):243-9.

16. Massey EK, Tielen M, Laging M, Beck DK, Khemai R, van Gelder T, et al. The role of goal cognitions, illness perceptions and treatment beliefs in self-reported adherence after kidney transplantation: a cohort study. J Psychosom Res. 2013;75(3):229-34.

17. Sgnaolin V, Figueiredo AEPL. Adesão ao tratamento farmacológico de pacientes em hemodiálise. J Bras Nefrol. 2012;34(2):109-16.

18. Jain D, Green JA. Health literacy in kidney disease: review of the literature and implications for clinical practice. World J Nephrol. 2016;5(2):147-51.

19. Instituto Brasileiro de Geografia e Estatística. PNAD Contínua 2016: $51 \%$ da população com 25 anos ou mais do Brasil possuíam apenas o ensino fundamental completo [acesso 2018 Jun 21]. Disponível em: https:// agenciadenoticias.ibge.gov.br/agencia-noticias/2013-agencia-de-noticias/releases/18992-pnad-continua-201651-da-populacao-com-25-anos-ou-mais-do-brasil-possuiam-apenas-o-ensino-fundamental-completo.html

20. Israni A, Dean C, Kasel B, Berndt L, Wildebush W, Wang CJ. Why do patients forget to take immunosuppression medications and miss appointments: can a mobile phone app help? JMIR Public Health Surveill. 2016;2(1):e15.

21. Diamantidis CJ, Ginsberg JS, Yoffe M, Lucas L, Prakash D, Aggarwal S, et al. Remote usability testing and satisfaction with a mobile health medication inquiry system in CKD. Clin J Am Soc Nephrol. 2015;10(8):1364-70.

22. Harrison TG, Wick J, Ahmed SB, Jun M, Manns BJ, Quinn RR, et al. Patients with chronic kidney disease and their intent to use electronic personal health records. Can J Kidney Health Dis. 2015;2:23.

23. Campbell J, Porter J. Dietary mobile apps and their effect on nutritional indicators in chronic renal disease: a systematic review. Nephrology. 2015;20(10):744-51.

24. Whitehead $L$, Seaton $P$. The Effectiveness of self-management mobile phone and tablet apps in long-term condition management: a systematic review. J Med Internet Res. 2016;18(5):e97.

25. Curran GM, Bauer M, Mittman B, Pyne JM, Stetler C. Effectiveness-implementation hydrid designs: combining elements of clinical effectiveness and implementation research to enhance public health impact. Med Care. 2012;50(3):217-26.

\section{Endereço para correspondência:}

Juliana Gomes Ramalho de Oliveira

Universidade de Fortaleza - UNIFOR

Programa de Pós-Graduação em Saúde Coletiva

Av. Washington Soares, 1321, Bloco S, Sala 1

Bairro: Edson Queiroz

CEP: 60.811-905 - Fortaleza - CE - Brasil

E-mail: julianagrdo@gmail.com 